\title{
Neil Schneiderman: «Die Aufgaben von Psychologen und Medizinern lassen sich nicht strikt voneinander trennen»
}

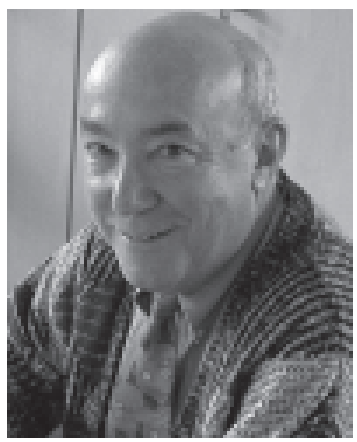

Prof. Dr. Neil Schneiderman ist Leiter der Abteilung für Gesundheitspsychologie und des Forschungszentrums für Verhaltensmedizin an der University of Miami, Florida, USA. Prof. Schneiderman gilt als einer der erfolgreichsten Wissenschaftler im Bereich der Verhaltensmedizin in den USA. Seine Forschung wird durch zahlreiche Drittmittelprojekte gefördert. Er ist Mitglied in vielen nationalen und internationalen Wissenschaftsorganisationen; unter anderem war er Präsident der "International Society of Behavioral Medicine» (1998-2000). Darüber hinaus ist Prof. Schneiderman in zahlreichen Fachzeitschriften tätig und war Herausgeber von «Health Psychology" (1984-1989) und dem "International Journal of Behavioral Medicine» (1994-1998). Das Gespräch mit inm führte Dipl.-Psych. Judith Heße, die sich 1999 für sechs Monate in seinem Forschungsinstitut aufhielt und dort in seinem HIV-Programm arbeitete.

Professor Schneiderman, Sie sind gerade in Deutschland, weil Sie den nächsten Kongress der International Society of Behavioral Medicine vorbereiten. Wann wird dieser Kongress stattfinden?

Neil Schneiderman: Der Kongress wird vom 25. bis 28. August 2004 in Mainz stattfinden. Er ist als Zusammentreffen vieler Wissenschaftler aus unterschiedlichen Gebieten zu verstehen und kann als Fortsetzung vieler erfolgreicher Treffen in den letzten 12 Jahren angesehen werden. Man kann diesen Kongress als Zeichen internationaler Zusammenarbeit und der Zusammenarbeit von Experten auf den Gebieten der Psychologie und Medizin sehen, wobei unter anderem die klinische Psychologie und Psychiatrie gut vertreten sein werden.
Was weckte Ihr Interesse an der Verhaltensmedizin?

Neil Schneiderman: Während meiner frühen Forschungsjahre war ich ursprünglich im tierexperimentellen Bereich anzusiedeln. Mein Hauptinteresse lag in der Grundlagenforschung über das ZNS und in der Neurobiologie von Konditionierungsvorgängen, die ich hauptsächlich an Kaninchen untersuchte. Eines Tages wurde ich auf einen Kongress zum «TypA-Verhalten» eingeladen. Ich war sehr überrascht und fragte mich, warum gerade ich eingeladen wurde. Ich hatte noch nie etwas von Typ-A-Verhalten gehört und arbeitete außerdem tierexperimentell. Mir wurde erklärt, dass es auf dem Kongress um bestimmte Verhaltensmuster gehe, die bei der Entstehung von Herzerkrankungen möglicherweise eine wichtige Rolle spielten. Ich sei eingeladen worden, weil man davon ausgehe, dass Tierstudien einen wichtigen Beitrag zur Erforschung dieser Verhaltensmuster liefern könnten.

Wie stellten Sie eine Verbindung zwischen Typ-A-Verhalten und Tierstudien her?

Neil Schneiderman: Während meines Vortrags führte ich das so genannte «active coping» bei Menschen aus und verglich es mit Verteidigungsreaktionen bei Tieren, die in bedrohlichen Situationen entweder weglaufen oder ihren Gegner angreifen. Dieses Verhalten geht mit einer gesteigerten Herzfrequenz und erhöhtem Blutdruck einher, da das autonome Nervensystem dafür sorgt, dass mehr Blut in die Muskeln gepumpt wird, um die erforderliche Verteidigungsreaktion ausführen zu können. Allerdings leben wir Menschen in einer Welt, in der es meistens nicht angebracht ist, auf sein Gegenüber loszugehen oder einfach wegzulaufen, wenn man sich bedroht fühlt. Dem autonomen Nervensystem können wir in solchen Stresssituationen natürlich nicht befehlen, sich ruhig zu verhalten, so dass ähnlich wie bei Tieren, die flüchten oder

\begin{tabular}{|c|c|c|}
\hline KARGER & (C) 2003 S. Karger GmbH, Freiburg & $\begin{array}{l}\text { Dipl.-Psych. Judith Heße } \\
\text { Karl-Marx-Str. } 94-96\end{array}$ \\
\hline $\begin{array}{l}\text { Fax +49761 } 4520714 \\
\text { E-mail Information@Karger.de } \\
\text { www.karger.com }\end{array}$ & $\begin{array}{l}\text { Accessible online at: } \\
\text { www.karger.com/ver }\end{array}$ & $\begin{array}{l}\text { D-54290 Trier } \\
\text { Tel. +49 } 651 \text { 201-3680 } \\
\text { Fax -3690 } \\
\text { E-mail hessej@uni-trier.de }\end{array}$ \\
\hline
\end{tabular}


angreifen, eine gesteigerte Aktivität des sympathischen Nervensystems mit erhöhtem Blutdruck, Steigerung der Herzfrequenz und vermehrter Katecholamin- und später Kortisolausschüttung zu beobachten ist. Im Laufe der Zeit stellte ich fest, dass die sympathische Aktivität bei Patienten mit Hypertonie zum Teil vergleichbar ist mit Reaktionen in ambivalenten, Angst einflößenden Situationen. In solchen Situationen weiß man oft nicht, wie man sich verhalten soll, ob man weglaufen oder still bleiben soll in der Hoffnung, der Angst einflößende Reiz werde bald von alleine weggehen. Eine interessante Beobachtung in diesem Zusammenhang ist, dass der erhöhte Blutdruck nicht primär von einer erhöhten Herzaktivität herzurühren scheint, sondern von einer Verengung der Blutgefäße und einem Anstieg im peripheren Widerstand.

\section{Wurde so Ihr Interesse geweckt, auch am Menschen zu forschen und dieses Wissen klinisch zu nutzen?}

Neil Schneiderman: Allmählich verlagerte sich mein Schwerpunkt vom tierexperimentellen Bereich auf das autonome Nervensystem und die Psychophysiologie. Ich untersuchte zunächst, welche bestimmten Verhaltensmuster zu Herzerkrankungen führen können. Natürlich spielen genetische Faktoren eine wichtige Rolle, z.B. die Vererbbarkeit von Hypertonie und Herzerkrankungen. Aber auch psychosoziale Eigenschaften wie Depressivität und feindseliges Verhalten und gesundheitliches Risikoverhalten, also Rauchen, Alkoholmissbrauch und Bewegungsmangel, können mit der Entstehung und dem Verlauf dieser Erkrankungen in Zusammenhang stehen. Wir fragten uns, ob der Beitrag bestimmter Verhaltensweisen zu der Entstehung und dem Verlauf von Herzerkrankungen über biologische und endokrine Veränderungen erklärt werden kann, und fanden bald Bestätigung für unsere Vermutung. Aus psychologischer Sicht stellte sich als nächstes die Frage, was für Interventionen benötigt werden, um das Risiko von Herzerkrankungen durch Verhaltensmodifikationen zu vermindern. Wir entwickelten Interventionsprogramme, die zum Beispiel das Ernährungsverhalten, körperliche Betätigung und den Umgang mit Stress veränderten, und fragten uns, welche Auswirkungen diese Interventionen auf subklinische Krankheitsmerkmale und natürlich auf den Krankheitsverlauf an sich hatten. Mit der Zeit erweiterten wir unsere Forschung auf andere Krankheitsbereiche, z.B. HIV/AIDS, und stellten uns auch hier die Frage, ob Verhaltensmodifikationen in Form von Entspannungsübungen, Sport, Bewertungsprozessen oder sozialer Unterstützung über endokrine Mechanismen den Krankheitsverlauf beeinflussen können. Wir stellten in unserer Forschungsarbeit also nicht nur die Frage, $o b$ Verhaltensmodifikationen Veränderungen im Krankheitsverlauf bewirken, sondern auch, über welche biologischen Mechanismen diese Veränderungen vermittelt werden.
Das von Ihnen gegründete Behavioral Medicine Research Center genießt international hohes Ansehen. Gibt es ein Geheimnis für diesen Erfolg?

Neil Schneiderman: Unser Behavioral Medicine Research Center kann als gemeinsames Forschungsinstitut von Psychologen und Medizinern verstanden werden; etwa die Hälfte der Mitarbeiter sind Psychologen, die andere Hälfte sind Mediziner. Unser Vorteil ist, dass wir keine institutsfremden Ärzte um Kooperation bei unseren Studien bitten müssen, sondern dass unsere Ärzte, die auch klinisch arbeiten, direkten Zugang $\mathrm{zu}$ Patienten haben und sie häufig für unsere Studien begeistern können. Im Großen und Ganzen lassen sich unsere Forschungsprojekte in verschiedene Gruppen unterteilen: Im Bereich der Herz-Kreislauf-Erkrankungen forschen wir bereits seit 1984/85. Ein Projekt in dieser Gruppe untersucht tierexperimentell genetische Determinanten von Arteriosklerose, in einem weiteren Projekt untersuchen wir Jugendliche mit erhöhtem Blutdruck und bieten ihnen Verhaltensmodifikationstrainings an, um das Risiko von Langzeitschäden bis hin zu Herzinfarkt zu verringern. Uns interessieren sowohl psychosoziale und behaviorale Aspekte, als auch endokrine und immunologische Faktoren. Dann haben wir mehrere HIV/AIDS-Studien. Abgestimmt auf die Bedürfnisse der Teilnehmer werden Stressbewältigungs- und Entspannungstrainings durchgeführt, in denen auch die Wichtigkeit der Medikamentencompliance betont wird. In einer Gruppe sind HIVpositive, homosexuelle Männer, in einer anderen infizierte Frauen aus sozial benachteiligten Schichten und in einer weiteren Gruppe drogenabhängige Männer, die sich im Laufe ihrer Sucht infiziert haben.

\section{Können Sie mir noch Näheres über Ihre Interventionsstudien erzählen?}

Neil Schneiderman: Wir bieten Interventionen nicht nur für HIV-Patienten und Personen mit Herzerkrankungen an, sondern auch für andere Patientengruppen. Prof. Dr. Michael Antoni, der bereits als Student bei uns gearbeitet hat, ist Leiter einer unserer Abteilungen, die sich mit Krebspatienten befasst. Eine seiner Studien ist z.B. auf Frauen mit Brustkrebs zugeschnitten, die in Kleingruppen Stressbewältigungs- und Entspannungsübungen lernen. Auch hier stellt sich uns wieder die Frage nach den Auswirkungen von Verhaltensmodifikationen auf endokrine und immunologische Variablen. Wir gehen davon aus, dass Hormone, vor allem Stresshormone, eine wichtige Rolle bei der Entstehung und dem Verlauf von Krankheiten spielen. Unser Kerngedanke ist demnach, mit Hilfe von Stressmanagement und Entspannungsübungen endokrine und immunologische Marker zu verändern, welche die Betroffenen anfällig für die jeweilige Erkrankung machen, und somit das Risiko für einen Rückfall bzw. ein schnelles Fortschreiten der Krankheit zu verringern. Ein weiteres Krebsprojekt, unter Leitung von Prof. Dr. Frank Penedo, be- 
fasst sich mit Männern mit Prostatakrebs. In einem auf diese Patientengruppe abgestimmten Interventionsprogramm werden relevante Themen angesprochen, die als Folge der Krankheit oder ihrer Behandlung auftreten. Viele Patienten leiden zum Beispiel unter Inkontinenz und Impotenz, was ihre Lebensqualität deutlich beeinträchtigt. In mehreren Therapiesitzungen versuchen wir mit Hilfe unserer bewährten Entspannungstechniken, sozialer Unterstützung und Zurückführung in den Alltag nicht nur die Lebensqualität zu verbessern, sondern wiederum biologische und immunologische Faktoren positiv zu beeinflussen. Dann gibt es noch eine Verhaltensinterventionsstudie für Patienten mit chronischem Erschöpfungssyndrom. Die Untersuchung steht unter der Leitung von Nancy Klimas, einer Immunologin, die sich auf Infektionskrankheiten spezialisiert hat und somit auch in unseren HIV/AIDS-Projekten die Hauptansprechperson für medizinische Fragen ist. Die Daten von all unseren Studien werden in einer großen Datenbank gespeichert. Da die Interventionen in den einzelnen Studien zwar auf die Belange der Patientengruppe abgestimmt sind, aber dennoch ähnliche Bausteine enthalten, ermöglicht uns diese gemeinsame Datenbank einen Zugriff auf Daten, die über das eigene Projekt hinausgehen. Das erleichtert natürlich einen Vergleich zwischen den verschiedenen Gruppen. Wir verfügen zudem über verschiedene Laborräume, so haben wir zwei Labore für die Analyse endokriner Daten, ein Immunologielabor und mehrere Labore für psychophysiologische und klinisch-physiologische Fragestellungen. Die Tatsache, dass sich bis auf wenige Ausnahmen alle wichtigen Arbeitsbereiche in einem Gebäudes befinden, vereinfacht die Kommunikation und erleichtert die interdisziplinäre Zusammenarbeit.

\section{Welche Möglichkeiten bieten sich in Ihrem Institut für Nachwuchswissenschaftler?}

Neil Schneiderman: In unserem Institut gibt es verschiedene Programme für Doktoranden und Post-Doktoranden, die vom National Institute of Health gefördert werden. Zu uns kommen einerseits Mediziner und Immunologen, die etwas über die Verhaltensmedizin und unsere Verhaltensinterventionen lernen wollen, andererseits Psychologen, die sich für klinische Fragestellungen interessieren. Die Fördergelder für Doktoranden werden fast ausschließlich für den Bereich der klinischen Gesundheitspsychologie zur Verfügung gestellt. Dabei ist es uns wichtig, den Forschungsbereich und die praktische Ausbildung nicht vollständig voneinander zu trennen, sondern beide Aspekte in die Ausbildung der Doktoranden zu integrieren. Für gewöhnlich dauert das Doktorandenstudium an unserem Institut 6 Jahre. Im ersten Jahr belegen die Doktoranden Seminare und Vorlesungen in Verhaltensmedizin, Psychoimmunologie, Physiologie und Statistik und beginnen mit der klinischen Ausbildung. Ab dem zweiten Semester arbeiten die Doktoranden unter enger Supervision 10 Stunden pro Woche mit Patienten. Im Laufe der Doktorandenzeit übernehmen die Studenten neben der Datenerhebung zunehmend verantwortungsvollere Aufgaben, wobei sich ihr Aufgabenfeld nicht unbedingt auf ihre eigenen Forschungsprojekte beschränkt. So kann es sein, dass ein Doktorand seine Dissertation in einem unserer kardiovaskulären Projekte schreibt, gleichzeitig aber Gruppenleiter innerhalb des Krebsprojekts ist und dort Interventionen anleitet. Zusätzlich lernen die Studenten unter Anleitung unterschiedliche Assays im Labor. Sie werden also in den verschiedensten Bereichen ausgebildet und schreiben zugleich ihre Doktorarbeit mit der übergeordneten Fragestellung, wie psychosoziale und biologische Variablen zusammenhängen.

\section{Wie würden Sie selbst Ihren Beitrag zur Verhaltensmedizin bezeichnen?}

Neil Schneiderman: Wenn ich den Worten anderer Leute und der American Psychological Association glauben darf, ist mein wichtigster Beitrag etwas, was heutzutage als selbstverständlich angesehen wird. Als ich anfing, mich für die Verhaltensmedizin zu interessieren, wurde auf der einen Seite Verhalten, und auf der anderen Seite wurden Erkrankungen betrachtet. Ich kann natürlich nicht behaupten, es sei allein mir zuzuschreiben, dass beide Seiten durch Berücksichtigung biologischer, endokriner Aspekte nicht länger getrennt voneinander betrachtet werden. Aber ich kann sagen, dass ich bei den Anfängen der interdisziplinären Forschung in diesem Bereich dabei war und dass ich vielleicht auch durch meine tierexperimentellen Arbeiten einen wichtigen Beitrag dazu geleistet habe. Man kann mich vielleicht als eine Art Katalysator zwischen Verhalten auf der einen und Krankheitsverlauf auf der anderen Seite verstehen. Ich bin daran interessiert, die Interaktion zwischen Stress, Biologie und Krankheit zu verstehen.

Einen weiteren wichtigen Beitrag würde ich in der Entwicklung von Interventionen sehen, mit denen gezeigt werden konnte, dass Verhaltensmodifikationen den Krankheitsverlauf beeinflussen können. Natürlich muss ich auch hier wieder betonen, dass auch viele andere Wissenschaftler in diesem Bereich wichtige Forschung betreiben. Wir haben hart dafür gearbeitet, von der Denkweise Abstand zu nehmen, die Aufgaben eines Psychologen und eines Mediziners strikt voneinander zu trennen. In unserem Forschungsbereich ist es wichtig, dass Psychologen biologische und immunologische Mechanismen verstehen und Mediziner Verhaltensweisen und psychosoziale Aspekte berücksichtigen. Ich bin sehr froh zu sehen, dass in Deutschland bereits vielerorts interdisziplinäre Forschung betrieben wird. Ich habe es seit meinem Einstieg in die Verhaltensmedizin stets als Aufgabe angesehen, die Wichtigkeit der Zusammenarbeit verschiedener Experten zu betonen, und habe auf Konferenzen regelmäßig versucht sicherzustellen, dass die Leute, die über Psychologie reden, wissen, worüber sie eigentlich reden, nämlich über Erkrankungen. 
Wie sehen Sie die Zukunft für Psychologen in der Verhaltensmedizin?

Neil Schneiderman: Um es bildlich darzustellen: Ich denke, die Tür hat sich geöffnet, aber als Psychologe muss man auch bereit sein hindurchzugehen. Psychologen müssen bereit sein, sich in die medizinische Nomenklatur und die Probleme im Gesundheitssystem einzuarbeiten, sonst werden sie nicht in der Lage sein, einen neuen Raum zu betreten. Wenn Psychologen im Bereich der Medizin, also in klinischem Umfeld auf dem Gebiet körperlicher und psychophysiologischer Erkrankungen arbeiten möchten, müssen sie sich natürlich auch auf diesem Gebiet einarbeiten. Lassen Sie mich die Entwicklung der letzten Zeit wie folgt darstellen: Vor einigen Jahren machten Ärzte noch Hausbesuche. Sie kannten ihre Patienten gut, denn sie wurden bei den unterschiedlichsten Beschwerden gerufen. Heutzutage geht man häufig zu einem Spezialisten, der aber vielleicht nur einen bestimmten Aspekt der Krankheit sieht. Nehmen wir zum Beispiel das metabolische Syndrom. Der eine Arzt diagnostiziert Bluthochdruck, der nächste Glukoseintoleranz usw. Früher hingegen erkannten die Ärzte wahrscheinlich mehr als ein Symptom, da sie den Patienten ja bei allen seinen Beschwerden aufsuchten. Das relativ junge Gebiet der Verhaltensmedizin ermöglicht es durch die ihr zugrunde liegende Denkweise nun wieder, bei Erkrankungen mehr als nur ein Symptom zu erkennen. Ob Arzt, Immunologe, Endokrinologe oder Psychologe, alle sollten fähig sein, Verhalten und Erkrankung in Beziehung zu setzen und angemessene Interventionen durchzuführen. Das soll nicht heißen, dass Psychologen unbedingt erlaubt werden muss, Medikamente zu verschreiben. Aber es sollte in Zukunft häufiger interdisziplinäre Teams geben, die sich gemeinsam und umfassend um die Bedürfnisse ihrer Patienten kümmern. Das Schlagwort für die Zukunft sollte also Kooperation, nicht Konkurrenz heißen. Es sollte ein gemeinsames Wissen aufgebaut werden, und es sollten Behandlungsformen entwickelt werden, die sowohl pharmakologische als auch Verhaltensmodule beinhalten und die für die Prävention und Behandlung solcher Krankheiten eingesetzt werden können, die auch auf Verhaltensveränderungen ansprechen.

Wir danken für das Gespräch und freuen uns auf ein Wiedersehen 2004 in Mainz! 